\title{
XXXVIII.
}

Aus dem Kgl. Institut für Infectionskrankheiten zu Berlin.

\section{Ueber die durch Suprarenin experimentell erzeugten Veränderungen.}

\author{
Von
}

\section{Dr. Julius Citron.}

(Hir 2 ligniren im Text)

Die hutdrueksteigerne Wirkung, die der non T'akanine zoerst rein dargestellen wirksamen substanz der Vebennicre eien ist, fülute dazu, Experimente in dor Richtung anzustollen, ob sich nicht künstliche Arterien-

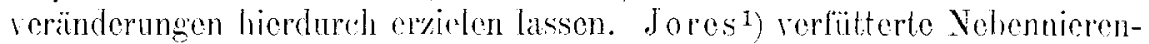
tabletten, olne indessen bei seinen Versuchsthieren positive Resullate crziclen zu können. Mit mohr Erfolg operirte Josué2), dem es clureh mehrwöchentliche intrarenöse Injection kleiner Dosen ven Idrenalin gelang, ausgedelintere arteriosklerotische Veränderungen zu erzeugen.

Diese Angaben, die zuerst mit einer gowissen Sliepsis aufgenommen wurden, sind inzwisthen mehrfach nachgeprüft und bestätigt worden.

So haben Erb jun. ${ }^{3}$ and Nissl, v. Rzentkowski4) and Fischer bei Kaninchen im Wesentlichen di gleichen Veränderungen gefunden.

Auf Teranlassung von Herrn Prof. Wassermann habe jeh seit dem Sommer 1904 zahlreiche Kaninchen mi intravenösen Suparenininjectionen behandelt. Dic Kaninchen erhiclien vicle Woehen hindureh täglich 3 bis 4 'Tropfen Suprarenini hydrochloric. 1: 1000 (Föchster Farbwerke) in die Ohrvene injicirt. Die ersten Dosen wurden ron den Thieren oft schlecht vertragen. Ls stellten sich Krömpfe, Jälhmungen, Zwangsbewegungen und oft acuter Tod ein, wobed einige Iale blutiger Scham aus rase und Mund trat. Indere Thiere dagogen, sogar kleine und wenig krältig

1) Jores, Wesen und Entwicklung der Artcriosklerose. Wiesbaden 1903.

2) Josué, Athérome aortique expérimental par injections répétées d'advénaline dans les reines. C. R. de la Sociéte do Biologie. 14. Nov. 1903.

3) Erb jun., 21. Congress f. innere Medicin in Leipzig. 1904.

4) v. Rzentkowski, Berl. kl. Wochenschr. 1904.

5) Fischer, Sitzung des psychiatrischen Vereins d. Rheinprovinz zu Bonn. 21. Nov. 1904. Autoreferat. Medic. Kl. 1905. 


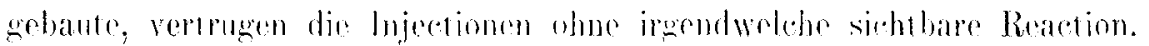
Sach kinzer Zeit bereits trat cine Gewohnnng an das Gift ein, so dass ich allmählich his aul mehr als das Yehnfache der Anfangsdosis gehen konnte. Da es sich bei dem Nebemnierengift un ein thierisches Gift handelt, welches freilish im Gegensal\% zu anderen Toxinen in seiner chemischen structur belannt ist, so bedarf die Frage weilerer Untersuchung. ob es sich bei dieser Gewohnung um cine active Lmmonisirung handeit, oder ob hier eine Frscheinung rorliegt, die in Inalogic zu dor Iorplin- und Arsenikgewöhnung zu setzen ist.

J)ie durch intravenöse Suprarenininjectionen erzielten Veränderungen waren im Wesentlichen conform der Bchandlungszeit. Ich konnte sowohl Aneurysmabildung, als auch Trübungen der drterienwandung, atheromatöse Geschwüre in der Aorta, Klappenveränderungen und Kalkablagerungen erzielen. In einem falle war eine ausserordentiche Herhypertrophie das hervorstechendste Merlimal. Das Herz war etwa doppelt so gross als normal.

Um diese Wirkung des Adrenalins resp. Suprarenios zu erklären, wiesen die ersten Autoren anf die hlutdruelisteigernde Eigenschaft desselben hin. Mllein diese lirklärung ist eine ungenügende. Das Adrenalin contfaltet im Organismus cine so vielgestaltige Wirkung, dass es nicht angängig ist, die Blutdrucksteigernng allein herauszugreifen, $1 \mathrm{~m}$ dic anatomischen Veränderungen der Arterien zu erklären.

Hierzu kommt, dass die Bludackisteigerung, wie Langlois ${ }^{1}$ ) und Battolli2) 1. A. nachgewiosen haben, schon nach maximal 3--4 Minuten voriibergeht.

Josucia) spricht sich in einer neneren Publication ebenfalls dahin aus, dass ausser der rermehrten Arterienspannng ein speciell schädigendes Monent da sein mïsse, da wiederholte Nieodingaben, welche gleidhfalls eine Bhtdruekstcigerung zur Folge lätten, keine Spur von Atheromatose cr\%eugten. Braun ${ }^{4}$ lat in einer vorläufigen Nittheilung über Versuche beriehtet, in denen er lianinehen eine Combination ron Adrenalin +.. Amylnitrit injicirte. Trotzdem die Blutdrucksteigerung ausblieb, soien arteriosklerotische lerändemugen cingetreten.

Andererseits werden durch die wirksime Substanz der Nebenniere sehr weituehende Sioffweehselstïrungen verursaled. Blumos, Zuclzers, und Hetzeeri). Looper und ('rouzons haben nachere-

1) Lianglois, These de la faculté des sciences de l'aris. 1897 .

2) Battelli, Société de biologie. 27. Dee. 1902. S. 956 .

3) Josuć, Paihogénie de l'athérome artériel. Le bulletín miéd. 1904. No. ss.

4) Braun, Sitzungsbericht der k. k. Gesellschaft der Aerzte zu Wien. Münch. med. Woch. 1905. No. 11.

5) F. Blum, Deutsch. Archir S. klin. Med. Bd. 71.

(6) Zuelzer, Berl. klin. Woch. 2. Jec. 1901.

7) Metzger, Münch. med. Woch. 25. März 1902.

8) Loeper und Crouzon, Arch. d. méd. expérim. 1904. 
wicken, dass 20 Minuten nach ciner intravenösen Injeetion von 6 Tropfen Adrenalin 1: 1000 cine Glykosurie eintrit, die 3,25-4,5 $\approx$ pro Liter ('rin betragen kann. Ed. Arousohn $n^{1}$, Richter ${ }^{2}$ ), EHJinger und Scelig ${ }^{3}$ ) haben die Beziehungen dieser Glykosuric zum Fieber und zu anderen Stoffwechselstörungen genar untersucht und haben interessante Zusammenlı̈̈nge aufgedeckt.

Während Zuelzer und Motzger dic Glykosurie auf eine temporäre Iyperglykämie zurïckführen, die ihren Lrsprung in einer steigerung der amylolytischen Thäigkeit der Leber (Zuelzer) lindet, sehen Jépine und Boulud ${ }^{*}$ ) das ursächliche lloment in einer Verminderung der inneren Pankreassecretion.

Gegen diese Annahme sprechen Versuche ron D. N. Paton ${ }^{5}$, der Idrenalinglykosurie auch bei Thicren erzeugte, denen das Pankreas entlernt worden war.

Loeper und Crouzon (l. c.) fanden fermer Verminderung der Lipase und Amylase, Verringerung der Zahl der Erythrocyten, Veränderungen an den blutbildenden Organen und Hypertrophie der Nebennieren.

Dem entspricht es, dass pathologische Veränderungen bei langdauernder Adrenalinbehandlung auch ausserhalb des Gefässsystems auftreten können. Das Phänomen der Glykosurie lenkte meine Aufmerksamkeit auf dic Leber, nnd ich land hier in dor That starke Veränderungen.

Zwei Typen lassen sich hier unterscheiden, indem die Veränderungen sowohl rom interstitiellen Bindegewobe als auch rom Parenchym ausgehen können. Nach wenigen Injectionen bereits zeigt das interstiticlle Bindegewebe, besonders wenn mit der Suprareninmenge schnell gestiegen wird, Neigung zur Wtcherung. Dis Leberläppchen treten dadurch doutlicher hervor, ohne dass zunächst das Parenchym selbst gesehädigt wird. Nur dic Staungserscheinungen seitens dor Vona centralis sind bemerkenswerth (s. Figur 1). Diese Veränderungen, die an cine beginnende Jcber(irrhose erinnern, sind bisher nicht genügend gewürdigt worden. v. Rzentkowski (l. c.) beschreibt zwar in seinen Protokollen ähnliche Bofunde. zieht aber keinerlei Schlüsse daraus. Noch sehwerer als die chen besehricbenen Veränderungen sind die des parenchymatösen Typus.

lin besonders charakteristiseher Fall betrifft ein Kaninchen, das in der Zcit rom Juni bis August 1904 täglich 0,2 Supraren. hydrochlor. $1: 1000$ intrarenös injicirt bekam. Die Injectionen wurden stets gut vertragen. Ende August wurden dic Injectionen ausgesetzt. Anfang October starh das Thier spontan. Die Section ergab mächtigen lseites (4-5 Liter Flïssigkeit), Hydrothorax und Hydropericard. Keine besondere Herzlypertrophie, eino rerkalkte Stelle in dem Jortenbogen, geringgradiges

1) Ed. Aronsolin, Virchow's Archiv. Bd. 174. 1908.

2) Kichter, Berl. klin. Wocb. 1903. No. $3 \pi$.

3) Ellinger u. Seelig, Münch. med. Woch. 1905. No. 11.

4) C. R. de l'Acad. d. Sciences. 12. Jan. 1903.

5) D. N. Paton, Journ. of Physiology. XXXIl. 1904. 
Aneurysma der Aorta thoraledea. starke Milzererösserung und auffallend kleine Teber. Die mikroskopisehe Lntersuehung der Leher zeigte an manchen Stellen stärkste Bindegewebswucherung. Das Bindegewebe war in dic lacberacini lineingewuehert und hatte klcine Parenohyminseln grebildet. In anderen Stellen dagegen war ron Bindegewehe fast niehts zu sehen. Mier behersehten die parenchymatösen Degenerationen durchaus das Bild (s. Fig. 2). Nur kleine Reste normalen Gewebes waren noch in der Peripherie vorhanden. Die Vena centralis zeigte nur mässige Staung. Diesc parenchymatöse Leberdegeneration ist zu einem Theil sicher Staungswirkung. andererseits lässt sich der Gedanke doch nicht von der Hand weisen, dass hicr noch eine specifiselı Sehädigung hinzu-

Fig. 1.

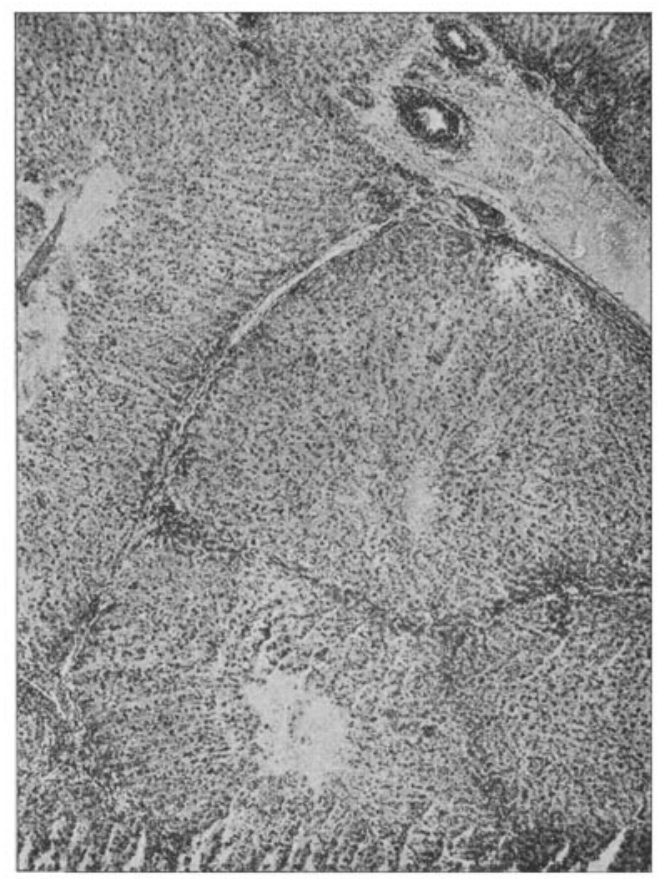

Figur 1. Kaninchenleberschnitt (Alliohol, Hämatoxylin-Eosinfürbung). 50 fache Vergrösserung. (Photogr. v. Prof. Zettnow). 12 intrarenöse Suprarenininjectionen zu $0,2 \mathrm{ccm} \mathrm{1/1000}$. Staungscrscheinungen. Interstitielle Bindegewebswucherung. Parenchym zeigt lieine stärkeren Veränderungen.

getommen ist, dass hier eine Giftwirkung vorlegt. langlois ${ }^{1}$ ) und Battediª haben in dieser Ilinsidet bemerkenswerbe Versuche angestellt. die zu dem Resultate fühten, dass dic Lober die Funetion hat.

1) Langlois, Sociéte de biologie. 29. Mai und 12. Juni 1897.

2) Battelli, Soc. de biolog. 13. Decemb. 1902. 
das Adrenalin seiner den Buddrak erhöhnden Eigenschaft zu berauben.

Andererscits wissen wir fon zahlrejehen Giften, dass sie in Stande sind, ähnliehe laberdegenerationen zu bewirken, so rom Phosphor, Alkohol, Chloroform, kohlensaurem Ammon, karbaminsaurem Ammon, Toluylendiamin u. a. Aueh die klinischen Erfabrungen spreehen dafür, dass zwischen Leber- and Arterienveranderungen ein gewisser ätiologischer Zusammenhang besteht, der wahrscheinlich in einer Yoxe zu suchen ist. welche wie das Syphilisvirus und der Alkohol sowohl auf die Leber als auch auf die Irtericnwandung spociliseh giftig wirlit. Veuce Untersuchungen laben andererseits aul die Häufigkeit der Arteriosklerose bei Glykosurie anfmerksam gemacht. Ledelor liegen bishor keine Beobachiungen

Fig. 2.

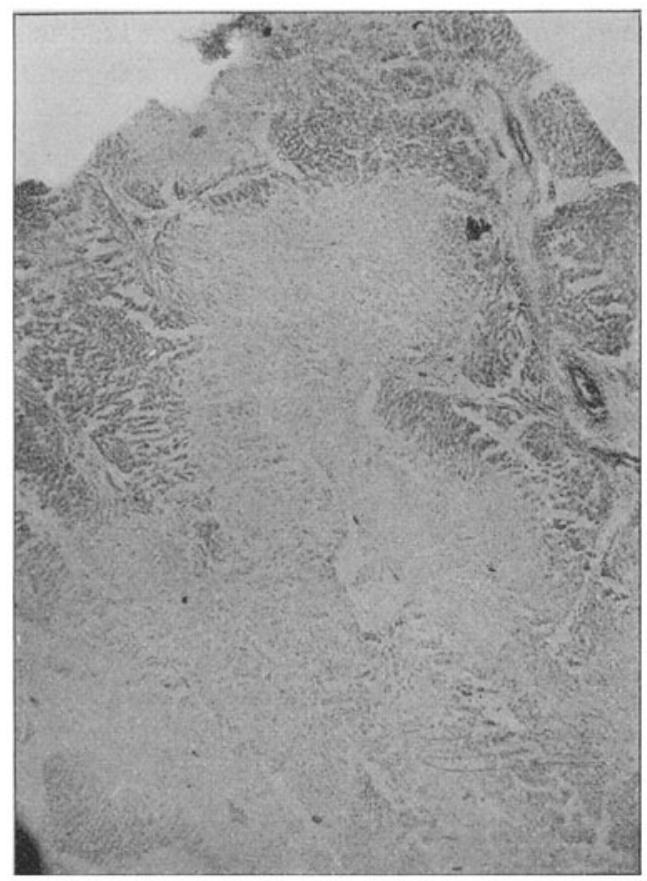

Figur 2. Káaninchenleberschnitt (Alkohol, llämatoxylin-Eosinfärbung). 40 fache Vergrösserung. (Photogr. ron P'rof. Zettnow.) :3 Monate hindurch tägl. intrarenöse Supratenininjectionen $z u \quad 0,2 \mathrm{ccm} 1 / 1000$. Geringe Stauung. Keine Bindegewebswucherung stärkeren Grades. Nahezu vollständige Degeneration des Jeberparenelyyms. Nur in den Randbezirken normales Jeberparenclym.

darïber ror, ob und eventucll in wie weit Vebemnierenveründerungen (abnorme Hypersectetion ron Nebennicrengift) für die Aetiologie dieser Trias, Arteriosklerose, Glykosurie und Leberirmose resp. -Atrophic, beim Jensehen in Erage kommen können. 
654 T. Citron, Ueber die durch Suprarenin experimentell erzeugten Veränderungen.

Ebenso wie Leberseränderungen durclu Gifte bewirkt werden künnen. ist dies auch experimentell ron den Arterienverïnderungen bewiesen worden. Aus den Arbeiten von (iilbert und Lion ${ }^{1}$, (Croca ${ }^{2}$, Thérese ${ }^{3}$ ), Pernices) and Boinet und Romary geht hervor; dass es dureh intrarenöse Injeetion ron Bakterien und Toxinen, sowic ron Harnsäure, Bleiund Quecksilberverbindungen gelingt, mikrostopische und geringgradige makroskopische lrterienläsionen hervomurufen. Welehe unrerkennlare Aelinlichkeit mit der Mrterienatheronatose aufweisen. Die Mirkung dieser Gifte kann wesentlieh gesleigert werden, wenn trammatische Verletzungen dor Arterienwandung hinzukommen. In diesen Fallen liaben die Autoren ausgedehnte Gesehwïre und Kalkahhigerungen beobathten können.

Diese Beobalehtungen erklären uns die Wirkmngsweise des Adrenilins ungezwungen dahin, dass in ihm die Combination der giftigen und der blutdruekstejgemden Gruppe den starken Lffeet bedingt. Die plötalide Blutdrucksteigerung setzt kleine nechanishe Läsionen der Arterienwandung und nun wirki das specifische Protoplasmagift destruirend cin.

1) Gilbert u. Lion, Artérites infectieuses (C. R. de la Société de Biolog. 22. Oct. 1889. - G. Lion, Essai sur la nature des endocardites (Thiese do Paris 1890.) -- Gilbert u. Lion, Note sur l'alhérome expérimental. (Archive de méd. expérim. 1904.)

2) Crocq, Contribution il l'etude experinent. Des artérites infectieuses. (Arch. de méd. expérin. 1894.)

3) 'Thérise, Thise de Paris $189 \%$ ?

4) Pernice, Ricerche intorno alla etiologia dell'arterite (Atti del R. Accal. Palermo 1895.)

5) Boinet u. Romary, Recherches expír. sur les aortites (Arch. de mód. exp. $189 \bar{i}$. 